Check for updates

Cite this: RSC Adv., 2017, 7, 20460

Received 20th December 2016 Accepted 1st April 2017

DOI: 10.1039/c6ra28440j

rsc.li/rsc-advances

\section{Effects of triphala and guggul aqueous extracts on inhibition of protein fibrillation and dissolution of preformed fibrils $\uparrow$}

\author{
S. N. Save and S. Choudhary (iD *
}

Herbal preparations have long been used for treatment of variety of diseases. However, a systematic and scientific evaluation of their effects and mechanisms of action have been lacking, although it is believed that synergistic complementation of the effects of the multiple chemical components in these preparations would be playing crucial regulatory roles in the different metabolic pathways which may interfere in a biological system. In this context, we investigate here protein fibrillation which is known to be an important process responsible for many neurodegenerative and other diseases. Using lysozyme as a model protein, we have studied using a combination of spectroscopic and microscopic techniques, the effects of two herbal preparations, derived from triphala and guggul, on the fibrillation process. We observed that both the extracts have the ability to inhibit protein fibrillation but triphala has much more dominating influence on fibrillation inhibition. The kinetics of fibrillation has been monitored by fluorescence spectroscopy as well as absorption spectroscopy, and the morphological changes associated with fibrillation have been monitored by transmission electron microscopy. Our experiments seem to suggest an interference (or synergy), though minor, between the effects of triphala and guggul on the fibrillation/aggregation process. We also observed that both triphala and guggul have the ability to dissolve preformed fibrils and aggregates of lysozyme, in a synergistic manner. We believe, the present indication of possible synergy between triphala and guggul can be conceptually extrapolated to the components of the individual preparations. Thus, the current work has significant therapeutic implications and will provide scientific basis to the development of new generation of phytopharmaceuticals which can be used alone or in combination with other drugs.

\section{Introduction}

Many polyherbal formulations have been reported to be very effective for the treatment of diseases like cancer, cardiovascular disorders, ophthalmic problems, liver dysfunction and many others. ${ }^{1-3}$ The use of herbal systems of medicine in combination with other therapeutic systems like chemotherapy and radiotherapy has also been documented..$^{4-6}$ Many phytomedicines are marketed as whole plant extracts containing multiple components. The strength of such a treatment is believed to be the synergy between the multiple components of the extracts, although experimentally it has been difficult to demonstrate this synergy because of the large number of components present in the extracts. Synergistic effects of drugs contribute to improved therapeutic effects due to some positive interactions and their complementary effects among their different components. However, a systematic and scientific

UM-DAE Centre for Excellence in Basic Sciences, University of Mumbai, Kalina Campus, Mumbai 400098, India. E-mail: sinjan.choudhary@cbs.ac.in

$\dagger$ Electronic supplementary information (ESI) available. See DOI: $10.1039 / \mathrm{c} 6 \mathrm{ra} 28440 \mathrm{j}$ evaluation of these effects and their mechanisms of action have been lacking. In this context, we report here the inhibitory and synergistic effects of triphala and guggul extracts on protein fibrillation.

Triphala is one of the age-old and well known polyherbal medicine of the 'Rasayana' group. 'Rasayana' drugs are integral part of Indian system of medicine (ISM) and are believed to promote immunity, health and longevity. ${ }^{7-9}$ Triphala is made of equi-proportion mixture of dried fruits of three plants: Terminalia chebula (Haritaki), Terminalia belerica (Bibhitaki), and Phyllanthus emblica or Emblica officinalis (Amalaki or the Indian gooseberry). ${ }^{10,11}$ This formulation is rich in antioxidants and strengthens the neurocrine, endocrine and immune systems of the body. Besides this, triphala is also reported to have antibacterial, antimalarial, antifungal, antiallergic, and antiviral activities. These properties of triphala evidently make it a suitable medicine to treat anaemia, jaundice, fever, cough and diarrhoea. ${ }^{12-14}$ Apart from all the above mentioned properties, triphala also possesses antioxidant, antimutagenic, antineoplastic, chemoprotective, radioprotective, and chemopreventive effects. Recent studies have demonstrated that triphala not only exerts protective effects on dermal fibroblasts and human 
keratinocytes ${ }^{15}$ but also mediates regulation of endothelial cell functions. ${ }^{15,16}$ In addition, another recent work it has been successfully demonstrated that aqueous and alcoholic extracts of triphala and their active compounds have potential to prevent epithelial to mesenchymal transition in retinal pigment epithelial cells. ${ }^{17}$

Another very effective herbal medicine is 'guggul' which is an oeolgum resin and is obtained from guggul tree Cammiphora mukul. ${ }^{3,18}$ Guggul has been used from centuries for the treatment of many diseases including hypercholesterolemia, rheumatism, arthrosclerosis, bone fractures, lipid disorders and obesity. ${ }^{4-7,19-22}$ Some studies have confirmed that guggul has been found to play a significant role in suppressing inflammatory and carcinogenic effects of many agents., ${ }^{\mathbf{8} 23}$ Guggul contains mixture of different guggul sterons which are reported to modulate numerous targets including growth factors, growth factor receptors, transcription factors, cytokines, enzymes, and genes regulating apoptosis. ${ }^{\mathbf{2 4 - 2 9}}$

Protein aggregation/fibrillation is associated with variety of diseases including Parkinson's and Alzheimer's diseases, cystic fibrosis, type 2 diabetes mellitus and others. A group of protein aggregation diseases also called as amyloidoses are characterized by deposition of fibrillar aggregates in affected tissues. ${ }^{30}$ Despite of having different structures and amino acid sequences of the proteins causing amyloidoses share same structural features. They are beta rich structures in which beta sheets are perpendicularly arranged to the fibrillar axis. ${ }^{31}$ These beta rich structures also share same tinctorial properties and bind with thioflavin $\mathrm{T}$ and Congo red dye. ${ }^{32,33}$

In spite of investing considerable efforts in searching for suitable therapeutic drugs against protein aggregation diseases, not much success has been achieved. Recently some of the natural plant products have been reported to have antiamyloidogenic and neuroprotective effects ${ }^{34-37}$ and there is possibility of existence of such type of other potent natural products. Our current study focuses on the unexplored features of triphala and guggul extracts on the inhibition of protein aggregation/fibrillation as well as on their probable synergistic effects; hen egg white lysozyme (HEWL) is taken as a model protein. HEWL is a 129 amino acid containing globular protein and is considered a suitable model for study due to its small size and availability of substantial structural information in the literature. ${ }^{38}$ This makes HEWL as a suitable model protein for study of protein stability, folding, misfolding and aggregation studies. ${ }^{39}$ It is reported that lysozyme can form fibrils under variety of conditions such as low $\mathrm{pH}$, high temperature, moderate concentration of guanidine hydrochloride ( $\mathrm{GuHCl}$ ) (2-5 M) at moderate temperature and alkaline $\mathrm{pH}$ at room temperature. ${ }^{40}$ Two naturally occurring variants of human lysozyme are responsible for autosomal hereditary systemic amyloidosis and are reported to be involved in formation and deposition of amyloid fibrils in various tissues. ${ }^{\mathbf{4 1}}$ This disease is linked with point mutations in the lysozyme gene, causing the non-conservative substitutions Ile56Thr or Asp67His, which make lysozyme less stable and amyloidogenic. ${ }^{42}$ This work represents a beginning of an elaborate exploration of herbal preparations as potential inhibitors of the fibrillation process.
The current work will provide a scientific basis to the development of new generation of phytopharmaceuticals which can be used alone or in combination with other drugs.

\section{Materials and methods}

\section{Chemicals}

Hen egg white lysozyme ( $>95 \%$ ), thioflavin T (dye content $0.65-$ 0.75), and guanidine hydrochloride (GuHCl, G3276) were purchased from Sigma-Aldrich Chemical Company (USA). All the solutions were prepared in Milli Q water from Merk Millipore. The experiments were performed in $60 \mathrm{mM}$ phosphate buffer at $\mathrm{pH}$ 7.4. The $\mathrm{pH}$ of solutions was adjusted using PICO+ Benchtop pH-meter (Labindia Instruments Pvt Ltd).

\section{Preparation of extracts}

Triphala and guggul tablets were obtained from Himalaya Pharmacy Ltd Company, India. A weighted amount of powder (2 $\mathrm{g} / 10 \mathrm{ml}$ ) from capsules was dissolved in Milli Q water at $60{ }^{\circ} \mathrm{C}$ and kept in the hot water bath for $2 \mathrm{~h}$. The solution was centrifuged it at $13000 \mathrm{rpm}$ at room temperature and supernatant was collected and filtered through $0.22 \mu \mathrm{m}$ filter. In order to obtain dry powders extracts were lyophilised using Labconco Free Zone 2.5 lyophilizer. Lyophilised powder was dissolved in phosphate buffer ( $\mathrm{pH}$ 7.4) and used for experiments.

\section{In vitro lysozyme amyloid formation}

The concentration of lysozyme was determined using Shimadzu UV-visible spectrophotometer, using an extinction coefficient $A_{1 \%}^{1} \mathrm{~cm}=26.5$ at $280 \mathrm{~nm}^{43}$ The samples of lysozyme at a concentration of $140 \mu \mathrm{M}$ were incubated at $37^{\circ} \mathrm{C}$ under stirring condition at $250 \mathrm{rpm}$ to induce fibrillation.

\section{Fluorescence spectroscopy}

The steady state fluorescence measurements were done on a Agilent fluorescence spectrophotometer. Kinetics of fibrillation was monitored using Thioflavin $\mathrm{T}$ (ThT) binding and $90^{\circ}$ light scattering assays. A stock solution of ThT was prepared in phosphate buffer (60 mM, pH 7.4). ThT, a cationic benzothiazole dye has been widely used to identify the amyloid fibrils having common structural features. ${ }^{\mathbf{4 4}, 45}$ The concentration of ThT was determined by using an extinction coefficient $E=$ $26620 \mathrm{M}^{1} \mathrm{~cm}^{-1}$ at $412 \mathrm{~nm} .{ }^{46}$ At different time intervals aliquot of incubated lysozyme solution was mixed with ThT solution such that the final concentrations of lysozyme and ThT for the fluorescence measurements were $4 \mu \mathrm{M}$ and $20 \mu \mathrm{M}$, respectively. The ThT molecules were selectively excited at $450 \mathrm{~nm}$ and emission was recorded at $482 \mathrm{~nm} \cdot{ }^{47}$ For all the measurements excitation and emission slit widths were fixed at $5 \mathrm{~nm}$. The reported fluorescence emission spectra of the complexes have been corrected by subtracting the reference spectra of the control solutions containing same amount of the dye. For $90^{\circ}$ light scattering experiments aliquots were taken out at different time intervals and diluted in phosphate buffer (60 mM, pH 7.4) such that the final concentration of the lysozyme was $4 \mu \mathrm{M}$. The excitation wavelength and emission wavelength were fixed at 
$350 \mathrm{~nm}$ for $90^{\circ}$ light scattering measurements. The obtained data from ThT fluorescence and $90^{\circ}$ light scattering measurements were satisfactorily fitted to the sigmoid curve represented by the following equation ${ }^{48}$

$$
Y(t)=Y_{\mathrm{i}}+m_{\mathrm{i}} t+\frac{Y_{\mathrm{f}}+m_{\mathrm{f}} t}{1+\mathrm{e}^{-\left[\left(t-t_{0}\right) / \tau\right]}}
$$

where $Y$ is the fluorescence intensity, $Y_{\mathrm{i}}$ and $Y_{\mathrm{f}}$ are initial and final fluorescence intensities, $t$ is time, and $t_{0}$ is the time to reach $50 \%$ of maximal fluorescence. The lag time is determined by $t_{0}-2 \tau$ where $\tau$ is the time constant of fibril growth and obtained by nonlinear regression. Each experiment was performed at least three times and an average was used.

For Congo red (CR) binding experiments aliquots of the lysozyme solution were taken out at different time intervals. The samples were diluted in phosphate buffer (60 mM, pH 7.4) such that the final concentrations of the lysozyme and CR were $4 \mu \mathrm{M}$ and $20 \mu \mathrm{M}$ respectively. After at least 10-12 minute incubation time absorption spectra were acquired from 400 to $700 \mathrm{~nm}$ using Shimadzu UV-visible spectrophotometer and cuvette with $1 \mathrm{~cm}$ path-length. The difference spectra were obtained by subtracting the spectra of CR alone and fibrils alone from those of the spectrum of CR and fibrils complex.

\section{Transmission electron microscopy}

The visualization of the amyloid fibrils was done on a JEOL JEM2100 Electron Microscope which operates at an accelerating voltage of $200 \mathrm{kV}$. The fibrils were prepared in the similar fashion as described earlier and then diluted to a final concentration of $20 \mu \mathrm{M}$ in order to prepare grids. The Formvarcoated 200 mesh copper grids were used for deposition of the samples. The negative staining of the samples was done with $2 \%$ aqueous uranyl acetate solution. After pre-rinsing with large volumes of water, a $0.22 \mathrm{~mm}$ filter was used to filter the stains. Uranyl acetate is known to produce high electron density and image contrast as well as impart fine grain to the image. ${ }^{49}$

\section{NMR spectroscopy}

NMR spectra of the aqueous extracts were recorded on a 500 MHz BRUKER Spectrometer. Water suppression was achieved by the standard Excitation Sculpting technique.

\section{Results and discussion}

\section{Spectroscopic signatures of extracts}

Several reports have appeared in the literature on the constituents of triphala and guggul. ${ }^{\mathbf{5 0 , 5 1}}$ These have relied mostly on mass spectrometric analyses. Though there will be slight variations depending upon the methods of extractions used, it appears that by and large there are more than 40 components in triphala and many of these are small molecules containing a large number of hydroxyl groups. We have recorded absorption and NMR spectra of our preparations as some sort of signatures. These are shown in Fig. S1 and S2 $\uparrow$ respectively. The absorption spectrum of triphala shows a well-defined peak and its absorption spectrum matches with an earlier report. ${ }^{52}$ The
NMR spectra of triphala and guggul extracts (Fig. S2 $\dagger$ ) are distinctive, although both have a high density of peaks in the 3$4 \mathrm{ppm}$ region. Triphala spectrum shows also weak peaks in the aromatic and methyl areas. We checked NMR signatures of triphala extracts from three other different sources, and they show significant resemblance [Fig. S3†]. A detailed analysis of these NMR spectra is neither intended nor practical at this moment because of the presence of large number of components in the preparations.

\section{Lysozyme fibril formation}

Fig. 1A shows the time course of fibrillation of lysozyme when incubated in presence of $4 \mathrm{M} \mathrm{GuHCl}$ at $\mathrm{pH} 7.4$ and $37{ }^{\circ} \mathrm{C}$ with stirring at $250 \mathrm{rpm}$. The fibril extension was monitored in the presence of ThT which interacts mainly with amyloid fibrils and gives characteristic emission maxima at $482 \mathrm{~nm}$ when excited at $450 \mathrm{~nm} .{ }^{47}$ The fibrillation curve shows sigmoidal behaviour consisting of three distinct phases; initial lag phase, a subsequent elongation phase and a final saturation phase. The lag time for lysozyme fibrillation was calculated using eqn (1) and is found to be $(1.92 \pm 0.05) \mathrm{h}$. The formation of lysozyme fibrils was also confirmed by transmission electron microscopy which shows network of lysozyme fibrils when the image was taken after $36 \mathrm{~h}$ of incubation (Fig. 1B).

\section{Effects of triphala and guggul on lysozyme fibrillation}

To see the inhibitory effects of triphala and guggul on lysozyme fibrillation ThT binding assay was carried out. Lysozyme solution was incubated at $37^{\circ} \mathrm{C}$ and in presence of $4 \mathrm{M} \mathrm{GuHCl}$ under stirring conditions at $250 \mathrm{rpm}$ in presence of different concentrations of triphala and guggul. Fig. 2 represents the fluorescence kinetics plots of lysozyme in absence and presence of different concentrations of triphala and guggul. In the absence of triphala (Fig. 2A), the increase in ThT intensity is not significant upto 3 hours, beyond which the intensity of ThT rises sharply up to 10 hours. After 10 hours lysozyme fibrillation seems to achieve saturation and ThT intensity does not show further change. In presence of $0.25 \mathrm{mg} \mathrm{ml}^{-1}$ triphala the ThT fluorescence intensity does not show significant increase even after many hours of incubation. Subsequent increase in the concentration of triphala to $0.50 \mathrm{mg} \mathrm{ml}^{-1}$ also show similar type of behavior (Fig. 2A). The plots exhibit lag phase kinetics and fitted well to the sigmoidal function described in eqn (1) $\left(R^{2}=\right.$ 0.995 to 0.985$)$. They also indicate that lysozyme follows nucleation-dependent polymerization model of aggregation. ${ }^{53}$ The lag periods of lysozyme fibrillation in presence of 0.25 and $0.50 \mathrm{mg} \mathrm{ml}^{-1}$ triphala are $(2.35 \pm 0.15) \mathrm{h}$ and $(2.38 \pm 0.40) \mathrm{h}$ respectively. Although there is only slight increase in the lag period of lysozyme fibrillation when triphala concentration was increased to $0.50 \mathrm{mg} \mathrm{ml}^{-1}$, the inhibitory effects on lysozyme fibrillation by triphala are clearly reflected in terms of maximum ThT fluorescence intensity. The maximum ThT fluorescence intensity represents the extent of fibrils formed and is significantly reduced when the concentration of triphala is increased from $0.25 \mathrm{mg} \mathrm{ml}^{-1}$ to $0.50 \mathrm{mg} \mathrm{ml}^{-1}$. The ThT fluorescence kinetic plot of lysozyme fibrillation in presence of 


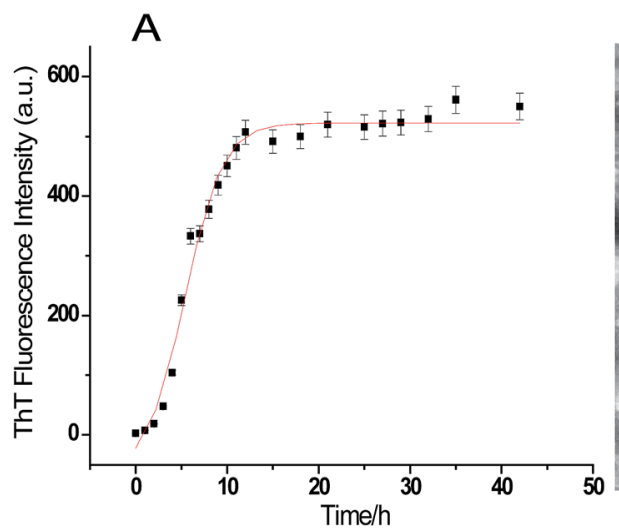

B

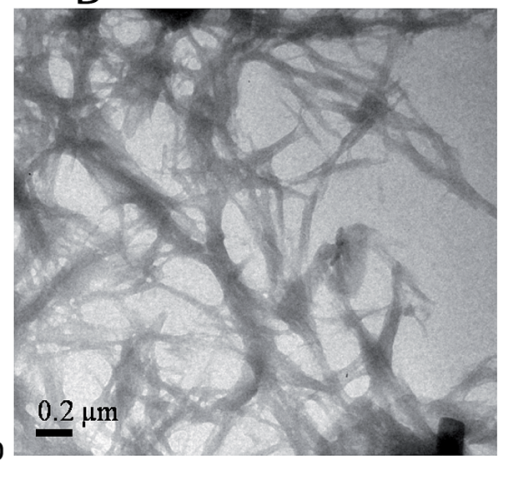

Fig. 1 (A) Kinetics of the lysozyme amyloid formation monitored by the binding of ThT with lysozyme amyloid fibrils, and (B) transmission electron microscopic (TEM) images of lysozyme fibrils after $36 \mathrm{~h}$ of incubation.
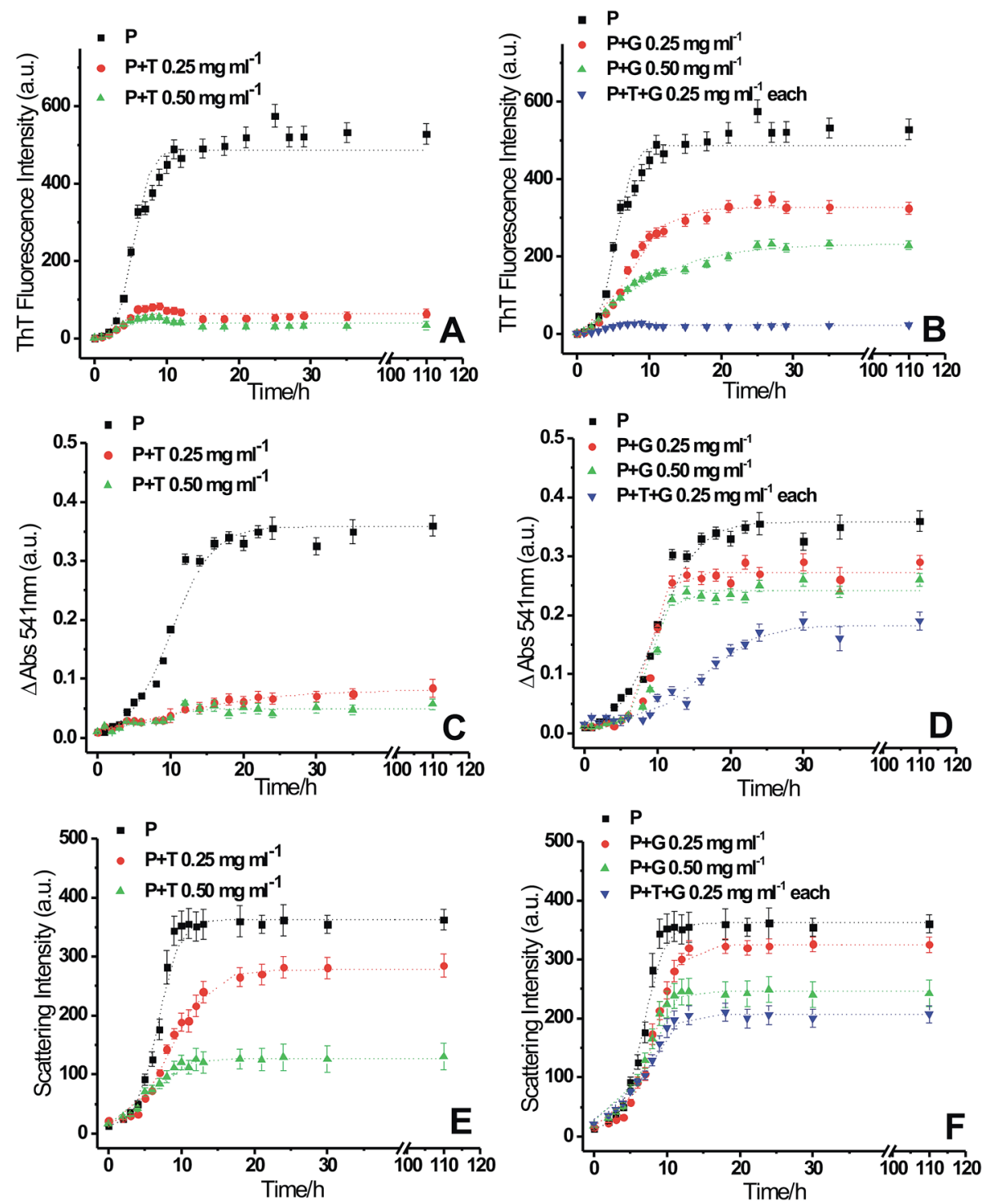

Fig. 2 Kinetics of lysozyme [P] fibril extension in absence and in presence of different concentration of triphala [T], guggul [G] and mixture of triphala and guggul $[T+G]$ studied by monitoring the changes in (A) and (B) ThT fluorescence emission intensity, (C) and (D) CR absorption at $541 \mathrm{~nm}$ and (E) and (F) $90^{\circ}$ light scattering respectively, as a function of time. 
$0.25 \mathrm{mg} \mathrm{ml}^{-1}$ guggul shows decrease in ThT fluorescence which further decreases when concentration of guggul is increased to $0.50 \mathrm{mg} \mathrm{ml}^{-1}$ (Fig. 2B). The lag time in presence of 0.25 and $0.50 \mathrm{mg} \mathrm{ml}^{-1}$ guggul are $(2.55 \pm 0.22) \mathrm{h}$ and $(2.01 \pm 0.10) \mathrm{h}$.

Lysozyme fibrillation and inhibition of fibrillation by triphala and guggul aqueous extracts were also confirmed by CR binding assay. As lysozyme fibrillation proceeds, an increase in absorbance of CR with more red shift was observed relative to that of the CR spectra alone or of native lysozyme. Increase in the absorbance of CR at $540 \mathrm{~nm}$ is characteristic of more CR binding to the fibrillar aggregates. ${ }^{54}$ Fig. $2 \mathrm{C}$ and $\mathrm{D}$ represent difference spectra plots of Congo red at $540 \mathrm{~nm}$ in absence and presence of different concentrations of triphala and guggul extracts. Decrease in the absorbance of CR at $540 \mathrm{~nm}$ in presence of triphala and guggul extracts in a concentration dependent manner well correlates with the ThT data and suggests that these herbal extracts inhibit lysozyme fibrillation.

A confusion may arise in the interpretation of fluorescence and absorbance spectral studies in the event of competitive binding of ThT/CR and triphala/guggul with the protein. Therefore, we also used an independent technique namely, $90^{\circ}$ light scattering which reports on molecular sizes. Fig. $2 \mathrm{E}$ and $\mathrm{F}$ represent light scattering plots of lysozyme in absence and presence of different concentrations of triphala and guggul. Here also, when the concentration of triphala and guggul were increased the scattering intensity decreased as a function of their concentrations. Similar to ThT assay, the lag time and amplitude were calculated for scattering plots using eqn (1) and are shown in Fig. 3. It is clear from Fig. 3A and B that the lag time increases when the concentrations of triphala and guggul are increased. On the other hand, amplitude decreases upon addition of triphala and guggul in a concentration dependent manner (Fig. 3C and D). ThT fluorescence detects fibrillar aggregates while light scattering detects both fibrillar as well as amorphous aggregates. ${ }^{55}$ The decrease in the amplitude while monitored by ThT binding and light scattering experiments clearly demonstrates that the extent of aggregation has decreased in presence of triphala and guggul. Fig. 3E and $\mathrm{F}$ show quantitatively the maximum ThT fluorescence and maximum scattering intensity respectively for free lysozyme and in the presence of different concentrations of triphala/guggul and their mixture. It is interesting to see that the maximum ThT fluorescence intensity in the presence of mixture of $0.25 \mathrm{mg} \mathrm{ml}^{-1}$ triphala and $0.25 \mathrm{mg} \mathrm{ml}^{-1}$ guggul is $(27 \pm 7)$ and that in the presence of $0.50 \mathrm{mg} \mathrm{ml}^{-1}$ triphala is (56 \pm 9 ). The difference between the two 29 is significantly more than the error bars. This is more so with regard to guggul. This observation seems to suggest possible synergy between the effects of the two herbal extracts. Since the same is not seen with CR, it appears that some other $\beta$-structures are detected by CR.

To see if there is synergy between triphala and guggul, ThT binding assay, CR binding assay and $90^{\circ}$ light scattering experiments for lysozyme fibrillation were carried out in presence of mixture of triphala and guggul $\left(0.25 \mathrm{mg} \mathrm{ml}^{-1}\right.$ of each component). The fluorescence kinetics profiles of lysozyme fibrillation monitored by ThT binding experiments (see Fig. 2B, blue line) clearly shows that the ThT fluorescence in presence of mixture of triphala and guggul is less compared to that when present individually $\left(0.50 \mathrm{mg} \mathrm{ml}^{-1}\right.$ triphala or $0.50 \mathrm{mg} \mathrm{ml}^{-1}$ guggul); it is marginally less in comparison to triphala but substantially less in comparison to guggul. When the same was monitored from CR binding assays mixture of $0.25 \mathrm{mg} \mathrm{ml} \mathrm{m}^{-1}$ triphala and guggul $\left(0.25 \mathrm{mg} \mathrm{ml}^{-1}\right)$ shows a somewhat different profile (see Fig. 2D, blue line) compared to that when they are present individually $\left(0.50 \mathrm{mg} \mathrm{ml}^{-1}\right)$. This may be due to presence of some $\beta$-structures in the solution which are not part of fibrils. The $90^{\circ}$ light scattering experiments for lysozyme fibrillation also show decrease in scattering intensity in presence of mixture of triphala and guggul (Fig. 2F).

For further verification of the results obtained from ThT and CR binding assays and light scattering experiments, transmission electron microscopy of lysozyme in presence triphala, guggul and mixture of the two was performed. Fig. 4A and $\mathrm{B}$ show transmission electron microscopic (TEM) images of lysozyme taken after $24 \mathrm{~h}$ of incubation when incubated at $37{ }^{\circ} \mathrm{C}$ under stirring condition at $250 \mathrm{rpm}$, in the presence of $0.50 \mathrm{mg}$ $\mathrm{ml}^{-1}$ triphala, and guggul respectively. It is evident from the TEM images that the amount of fibrils formed has considerably decreased in presence of triphala (Fig. 4A). Further, the number of fibrils formed is also less, and they are thinner and much smaller than those formed in the absence of triphala (Fig. 1B). The lysozyme fibrils formed in presence of guggul are small and thick and show bundle like morphology (Fig. 4B).

The TEM image of lysozyme fibrils in presence of triphalaguggul mixture shows clusters of small fibrils (Fig. 4C) which are similar to those observed in presence of triphala (Fig. 4A). This clearly demonstrates the dominating influence of triphala over guggul on the protein fibrils.

\section{Structural changes during lysozyme fibrillation in presence if triphala and guggul extracts}

Protein fibrillation is accompanied by huge conformational changes where different types of secondary structural elements are converted into $\beta$-sheet rich structure. Fig. $\mathrm{S} 4 \mathrm{~A} \dagger$ shows the farUV CD spectra for control experiments showing secondary structures of native lysozyme in absence and presence of different concentrations of triphala, guggul and their mixture. Fig. $\mathrm{S} 4 \mathrm{~B} \dagger$ shows the structural transition of $\mathrm{GuHCl}$ treated lysozyme from $\alpha$-helix rich structure to $\beta$-sheet rich fibrils as the fibrillation proceeds. Fig. 5A represents $\mathrm{CD}$ graph at $0 \mathrm{~min}$ of the fibrillation process. It is clear from the figure that lysozyme alone and lysozyme in presence of $0.50 \mathrm{mg} \mathrm{ml}^{-1}$ triphala, $0.50 \mathrm{mg} \mathrm{ml}^{-1}$ guggul and in the mixture of the two $\left(0.25 \mathrm{mg} \mathrm{ml}^{-1}\right.$ each) have almost similar kind of secondary structure. As the fibrillation proceeds (after $7 \mathrm{~h}$ of incubation) it is observed that a single minimum has appeared at $\sim 220 \mathrm{~nm}$ in lysozyme sample (in absence of any herbal extract) which is a characteristic of the presence of $\beta$-sheets (Fig. 5B, black line), where as in presence of $0.50 \mathrm{mg} \mathrm{ml}{ }^{-1}$ triphala it still has sufficient $\alpha$-helical content (Fig. 5B, red line). On the other hand in presence of $0.50 \mathrm{mg}$ $\mathrm{ml}^{-1}$ guggul $\beta$-sheets have started appearing (Fig. 5B, green line). But if we see the CD spectra of lysozyme in presence of mixture of triphala and guggul $\left(0.25 \mathrm{mg} \mathrm{ml}^{-1}\right.$ each), it has significant $\alpha$-helical content (Fig. 5B, blue line) and resembles 

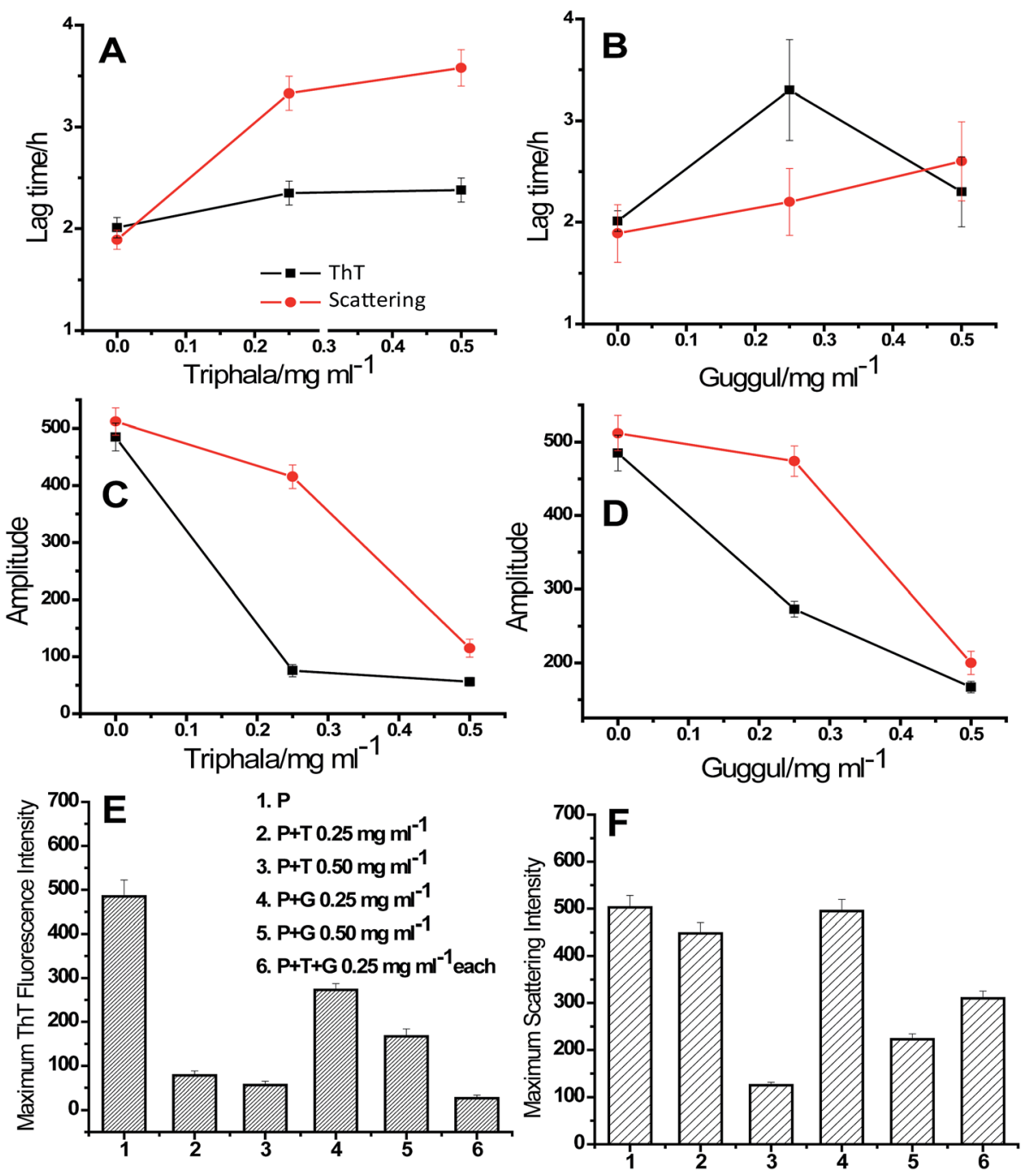

Fig. 3 (A) and (B) Lag time, (C) and (D) amplitude, (E) maximum ThT fluorescence intensity and (F) $90^{\circ}$ light scattering intensity of lysozyme [P] fibril formation in absence and presence of different concentrations of triphala, guggul and mixture of triphala and guggul.

that in presence of $0.50 \mathrm{mg} \mathrm{ml}^{-1}$ triphala. Fig. $5 \mathrm{C}$ represents the $\mathrm{CD}$ spectra recorded after $36 \mathrm{~h}$ of fibrillation process. It is clear from the figure that after $36 \mathrm{~h}$ of fibrillation $\beta$-sheets are formed in all the cases but the extent of $\beta$-sheets formation is different for different cases. In absence of herbal extracts lysozyme shows most negative ellipticity at $\sim 220 \mathrm{~nm}$ followed by that of in presence of $0.50 \mathrm{mg} \mathrm{ml}^{-1}$ guggul. In presence of $0.50 \mathrm{mg} \mathrm{ml}^{-1}$ triphala and in mixture of triphala and guggul $\left(0.25 \mathrm{mg} \mathrm{ml}^{-1}\right.$ each) the ellipticity is less. This suggests that although the fibrils have formed in each case but in presence of triphala extracts $\left(0.50 \mathrm{mg} \mathrm{ml}^{-1}\right)$ the extent of $\beta$-sheets formation is less. In presence of mixture of triphala and guggul the $\beta$-sheets formation is lesser than in presence of guggul alone $\left(0.50 \mathrm{mg} \mathrm{ml}^{-1}\right)$ and comparable to that in presence of triphala alone. It is clear from the $\mathrm{CD}$ data that presence of herbal extracts prevents fibrillation by inhibiting/delaying formation of $\beta$-sheets rich structures.
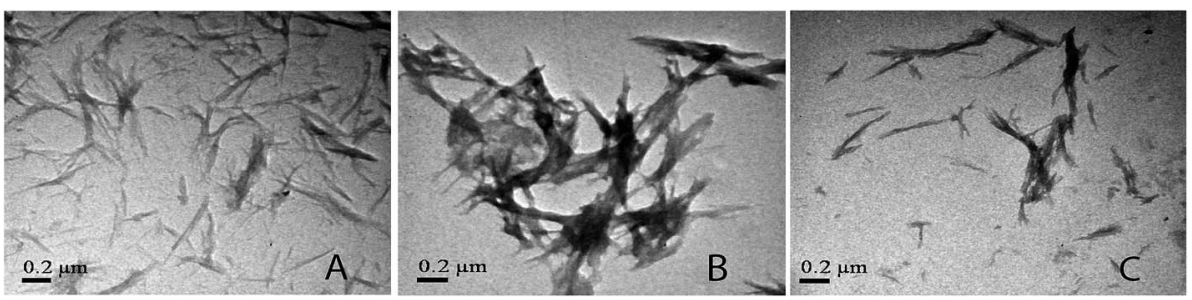

Fig. 4 TEM images of lysozyme after $36 \mathrm{~h}$ of incubation in presence of (A) $0.5 \mathrm{mg} \mathrm{ml}^{-1}$ triphala, (B) $0.5 \mathrm{mg} \mathrm{ml}^{-1}$ guggul and (C) mixture of triphala and guggul (0.25 $\mathrm{mg} \mathrm{ml}^{-1}$ each). 


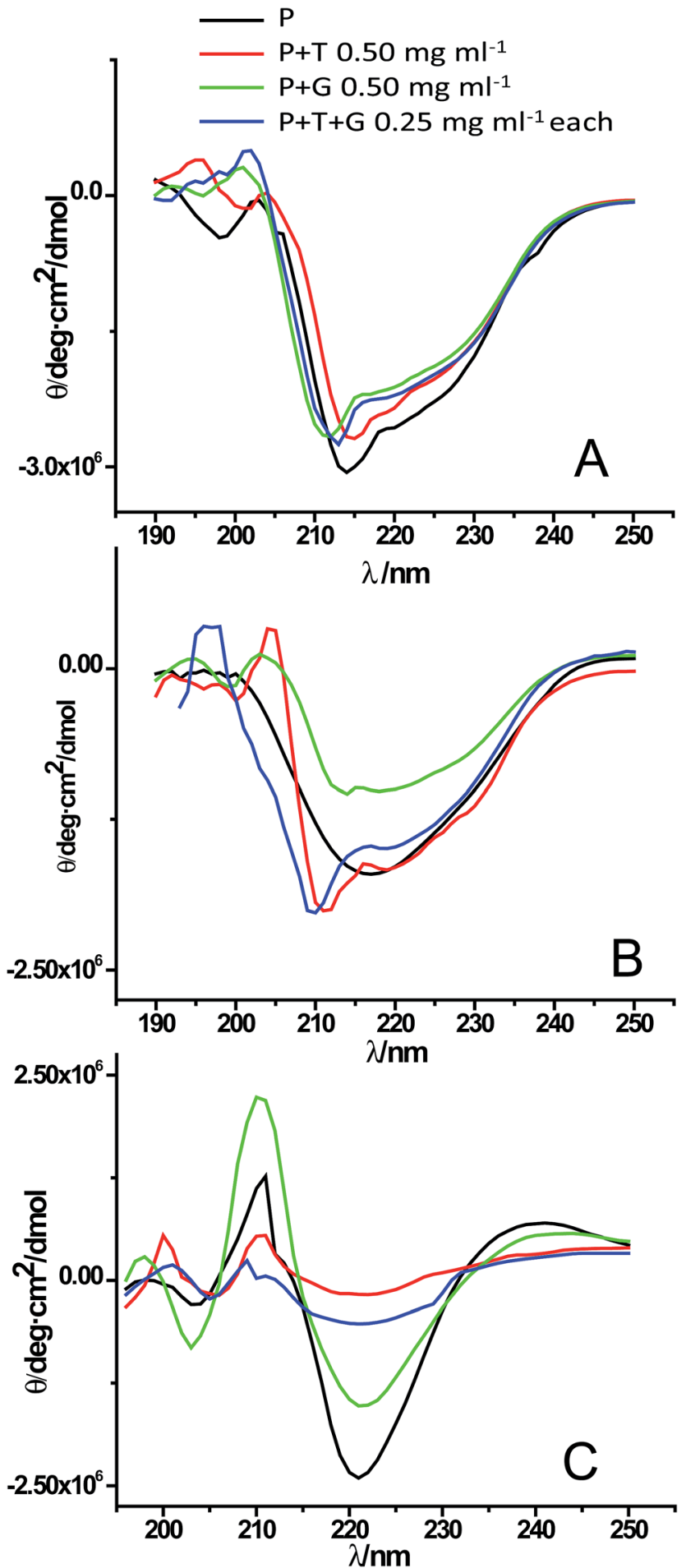

Fig. 5 Far UV-CD spectra of lysozyme [P] in absence and presence of triphala $[T]$, guggul $[G]$ and mixture of triphala and guggul $[T+G]$ at $(A)$ $0 \mathrm{~min},(\mathrm{~B}) 7 \mathrm{~h}$ and $(\mathrm{C}) 36 \mathrm{~h}$ of the fibrillation process.

Dissolution of lysozyme fibrils by triphala, guggul and their mixture

In order to check the possibility of triphala and guggul to dissolve lysozyme fibrils, ThT binding assay and light scattering experiments were performed. The lysozyme fibrils were prepared similarly as described earlier. The fibrils $(140 \mu \mathrm{M})$ were incubated in presence of different concentrations of triphala, guggul and their mixture and the ThT and light scattering intensities were recorded at different time intervals. The concentration of lysozyme aggregates was kept $3 \mu \mathrm{M}$ for both ThT binding and light scattering experiments. Fig. 6A and B show ThT and scattering intensities plots of lysozyme fibrils, respectively in absence and presence of different concentrations of triphala, guggul and their mixtures as a function of time. It is evident from the figure that presence of triphala, guggul and their mixture has decreased the ThT fluorescence intensity significantly while the decrease in scattering intensity is not to that extent. Since ThT intensity is sensitive to fibrils, and not so much to amorphous aggregates which could also be present in the solution, it can be inferred that the presence of these herbal medicines has dissolved the lysozyme fibrils. On the other hand, the scattering data which reflects on molecular dimensions of the species present, suggests that perhaps amorphous
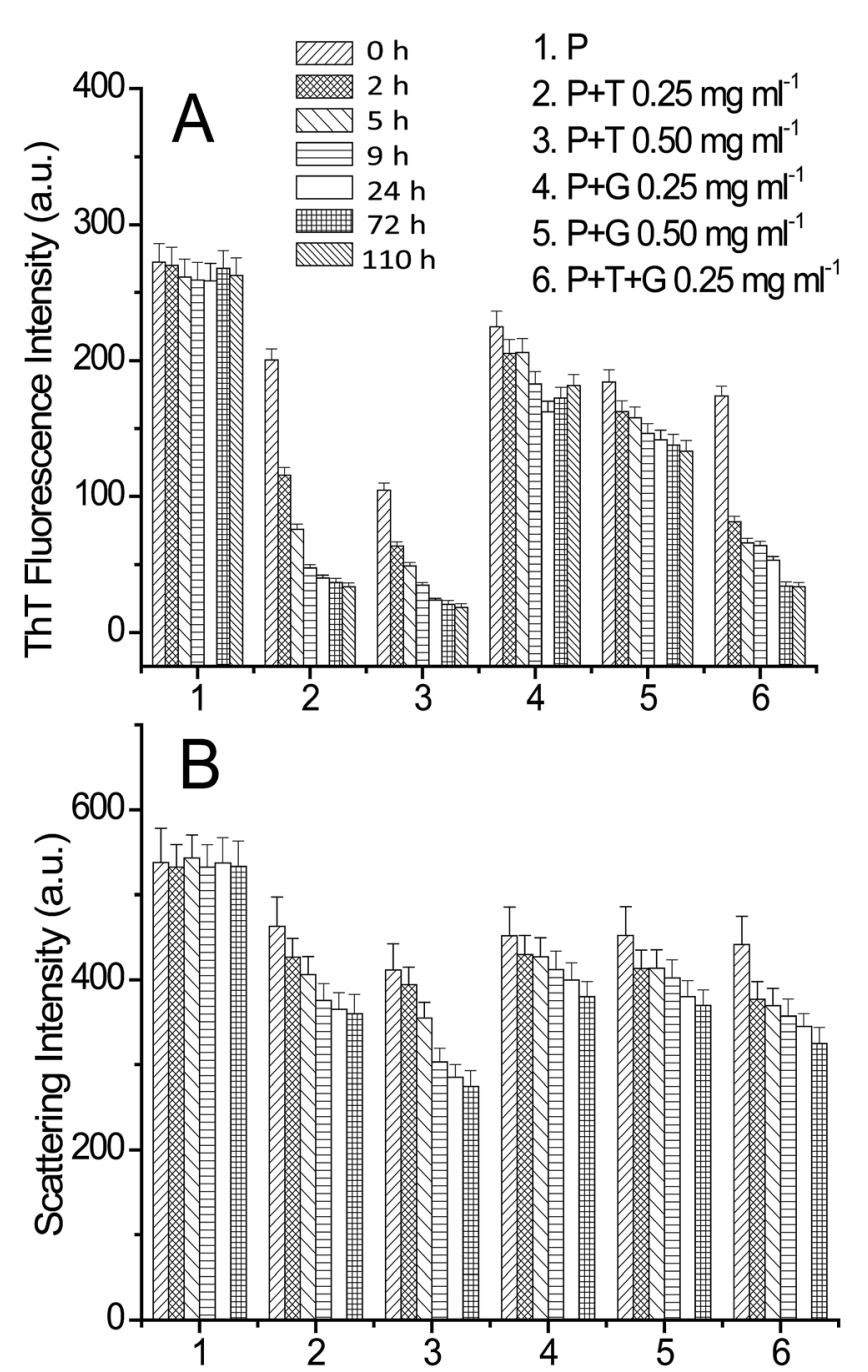

Fig. 6 Dissolution of lysozyme [P] fibrils in absence and presence of triphala $[T]$, guggul [G] and mixture of triphala and guggul $[T+G]$ monitored by (A) ThT binding assay and (B) light scattering experiment as a function of time. 


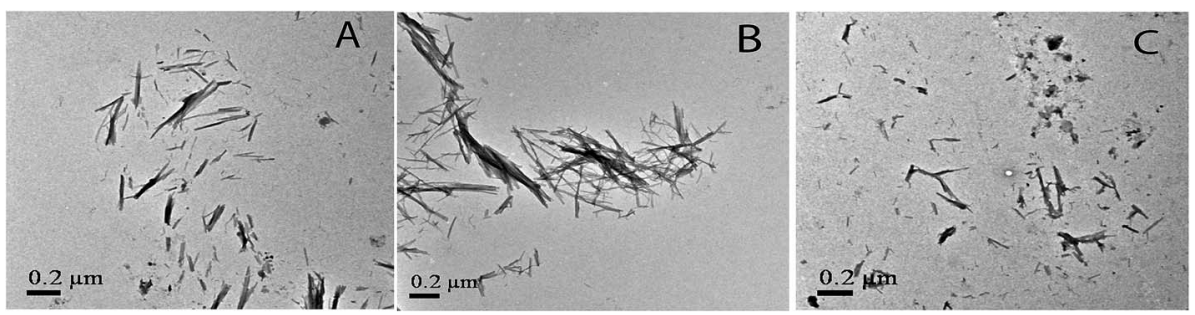

Fig. 7 TEM images of lysozyme fibrils after $36 \mathrm{~h}$ of incubation in presence of (A) $0.50 \mathrm{mg} \mathrm{ml}^{-1}$ triphala, (B) $0.50 \mathrm{mg} \mathrm{ml}^{-1} \mathrm{guggul}$ and (C) mixture of triphala and guggul $\left(0.25 \mathrm{mg} \mathrm{ml}^{-1}\right.$ each).

aggregates are affected less by these herbal medicines and in presence of these medicines some of the fibrils have been converted into smaller fibrils/amorphous aggregates resulting in only a small change in the scattering intensity.

For further verification of above findings, TEM imaging was performed for the dissolution of lysozyme fibrils. Fig. 7 shows TEM images of lysozyme fibrils incubated in presence of $0.50 \mathrm{mg} \mathrm{ml}^{-1}$ triphala, $0.50 \mathrm{mg} \mathrm{ml}^{-1}$ guggul and mixture of both $\left(0.25 \mathrm{mg} \mathrm{ml}^{-1}\right.$ each) for $72 \mathrm{~h}$. It is clear from Fig. $7 \mathrm{~A}$ that in presence of $0.50 \mathrm{mg} \mathrm{ml}^{-1}$ triphala, long thick fibrils have been broken/dissolved in smaller and thinner fibrils. In presence of $0.50 \mathrm{mg} \mathrm{ml}^{-1}$ gugguls fibrils have been dissolved but to a lesser extent compared to that in the presence of $0.50 \mathrm{mg} \mathrm{ml}^{-1}$ triphala (Fig. 7B). A mixture of broken fibrils like structure and amorphous aggregates are seen in TEM image of lysozyme fibrils in presence of triphala and guggul $\left(0.25 \mathrm{mg} \mathrm{ml}^{-1}\right.$ each) (Fig. 7C). This clearly demonstrates that the above described herbal medicines are not only capable of inhibiting lysozyme fibrillation but also of dissolving preformed fibrils effectively.

A thorough literature search suggests that some of the components (e.g. chebulagic acid, ascorbic acid etc.) of triphala have neuroprotective effects. ${ }^{56-58}$ The present work represents an elaborate exploration of the potential of some chosen herbal extracts in inhibition of protein fibrillation. We believe that it would also have implications for finding cures for protein fibrillation associated diseases. Knowledge of the binding parameters of the individual components of triphala and guggul with lysozyme during the fibrillation process can further provide insights into a detailed understanding of the mechanism of inhibition of fibrillation of lysozyme by these herbal preparations. However, this requires isolation and purification of the components of triphala and guggul and studying their effects individually and in combination (to check possible synergy). This is clearly, an extensive exercise which will be addressed in due course.

\section{Conclusions}

A combination of spectroscopy and microscopy has demonstrated the potential of herbal medicines triphala and guggul in prevention of lysozyme fibrillation. The use of ThT as a fluorescent probe and $\beta$-structure specific probe $\mathrm{CR}$ along with the $90^{\circ}$ light scattering experiments has successfully enabled elucidation of the extent of fibril formation in the protein solution. Triphala is observed to be a stronger inhibitor of fibrillation of protein than guggul. The data suggest some possibility of synergistic action of triphala and guggul on fibrillation of proteins. The results have successfully demonstrated that triphala, guggul and their mixture have potential to dissolve preformed fibrils.

\section{Author contributions}

Conceived and designed the experiments: SC. Performed the experiments: SNS SC. Analyzed the data: SC. Contributed reagents/materials/analysis tools: SC. Wrote the paper: SNS SC.

\section{Acknowledgements}

The authors gratefully acknowledge Prof. R. V. Hosur for his valuable help, suggestions and discussion during the course of this work. The authors would also like to thank Prof. S. M. Chitre for his support and encouragement. DST-SERB (SB/FT/ CS-169/2013), New Delhi is appreciatively acknowledged for financial assistance. The authors are thankful to Indian Institute of Technology Bombay, Mumbai for providing Transmission Electron Microscopic facilities and Tata Institute for Fundamental Research, Mumbai for NMR facilities.

\section{References}

1 P. Jadiya, A. Khan, S. R. Sammi, S. Kaur, S. S. Mir and A. Nazir, Biochem. Biophys. Res. Commun., 2011, 413, 605610.

2 V. P. Govindan, A. N. Panduranga and K. P. Murthy, Biochem. Biophys. Rep., 2016, 5, 359-364.

3 M. S. Baliga, J. Altern. Complement. Med., 2010, 16, 13011308.

4 V. T. DeVita, T. S. Lawrence, S. Hellman and S. A. Rosenberg, Cancer: Principles \& Practice of Oncology, Lippincott Williams \& Wilkins, Philadelphia, 8th edn, 2004.

5 E. J. Hall, Radiobiology for the radiologist, Lippincott, Williams \& Wilkins, Philadelphia, 5th edn, 2005.

6 E. M. Williamson, Phytomedicine, 2001, 8, 401-409.

7 S. Ponnusankar, S. Pandit, R. Babu, A. Bandyopadhyay and P. K. Mukherjee, J. Ethnopharmacol., 2011, 133, 120-125.

8 G. C. Jagetia, M. S. Baliga, K. J. Malagi and M. S. Kamath, Phytomedicine, 2002, 9, 99-108.

9 T. Sandhya, K. M. Lathika, B. N. Pandey and K. P. Mishra, Cancer Lett., 2006, 231, 206-214. 
10 C. P. Thakur, B. Thakur, S. Singh, P. K. Sinha and S. K. Sinha, Int. J. Cardiol., 1988, 21, 167-175.

11 M. S. Baliga, S. Meera, B. Mathai, M. P. Rai, V. Pawar and P. L. Palatty, Chin. J. Integr. Med., 2012, 18, 946-954.

12 R. Mathur, A. Sharma, V. P. Dixit and M. Varma, J. Ethnopharmacol., 1996, 50, 61-68.

13 K. J. Jeena, K. L. Joy and R. Kuttan, Cancer Lett., 1999, 136, 11-16.

14 P. K. Mukherjee, S. Rai, S. Bhattacharya, P. K. Debnath, T. K. Biswas, U. Jana, S. Pandit, B. P. Saha and P. K. Paul, Iran. J. Pharmacol. Ther., 2006, 5, 51-54.

15 S. R. Varma, T. O. Sivaprakasam, A. Mishra, L. M. S. Kumar, N. S. Prakash, S. Prabhu and S. Ramakrishnan, PLoS One, 2016, 11(1), e0145921.

16 S. Sivasankar, R. Lavanya, P. Brindha and N. Angayarkanni, PLoS One, 2015, 10(3), e0120512.

17 K. Lu and S. Basu, Sci. Rep., 2015, 5, 9642.

18 C. J. Sinal and F. J. Gonzalez, Trends Endocrinol. Metab., 2002, 13, 275-276.

19 M. L. Gujral, K. Sareen, K. K. Tangri, M. K. Amma and A. K. Roy, Indian J. Physiol. Pharmacol., 1960, 4, 267-273.

20 J. N. Sharma, Arzneimittelforschung, 1977, 27, 1455-1457.

21 R. Deng, Cardiovasc. Drug Rev., 2007, 25, 375-390.

22 H. Ichikawa and B. B. Aggarwal, Clin. Cancer Res., 2006, 12, 662-668.

23 S. Shishodia and B. B. Aggarwal, J. Biol. Chem., 2004, 279, 47148-47158.

24 A. DeGottardi, J. M. Dumonceau, F. Bruttin, A. Vonlaufen, I. Morard, L. Spahr, L. Rubbia-Brandt, J. L. Frossard, W. N. Dinjens, P. S. Rabinovitch and A. Hadenque, Mol. Cancer, 2006, 5, 48.

$25 \mathrm{~J} . \mathrm{Wu}$, C. Xia, J. Meier, S. Li, X. Hu and D. S. Lala, Mol. Endocrinol., 2002, 16, 1590-1597.

26 X. Ding and J. L. Staudinger, J. Pharmacol. Exp. Ther., 2005, 314, 120-127.

27 E. Owsley and J. Y. Chiang, Biochem. Biophys. Res. Commun., 2003, 304, 191-195.

28 N. Lv, M. Y. Song, E. K. Kim, J. W. Park, K. B. Kwon and B. H. Park, Mol. Cell. Endocrinol., 2008, 289, 49-59.

29 S. Shishodia, G. Sethi, K. S. Ahn and B. B. Aggarwal, Biochem. Pharmacol., 2007, 74, 118-130.

30 F. Rousseau, J. Schymkowitz and L. Serrano, Curr. Opin. Struct. Biol., 2006, 16, 118-126.

31 M. Sunde, L. Serpell, M. Bartlam, P. Fraser, M. Pepys and C. F. Colin, J. Mol. Biol., 1997, 273, 729-739.

32 R. Khurana, C. Coleman, C. Ionescu-Zanetti, S. A. Carter, V. Krishna, R. K. Grover and S. Singh, J. Struct. Biol., 2005, 151, 229-238.

33 D. B. Carter and K. C. Chou, Neurobiol. Aging, 1998, 19, 3740.

34 F. Mohammadi, A. Mahumudian, M. Moeeni and L. Hassani, RSC Adv., 2016, 6, 23148-23160.
35 R. V. Rao, O. Descamps, V. John and D. E. Bredesen, Alzheimer's Res. Ther., 2012, 4, 22.

36 L. Calcul, B. Zhang, U. K. Jinwal, C. A. Dickey and B. J. Baker, Future Med. Chem., 2012, 4, 1751-1761.

37 M. D. da Rocha, F. P. D. Viegas, H. C. Campos, P. C. Nicastro, P. C. Fossaluzza, C. A. M. Fraga, E. J. Barreiro and C. Viegas, CNS Neurol. Disord.: Drug Targets, 2011, 10, 251-270.

38 S. Choudhary and N. Kishore, PLoS One, 2014, 9(8), e104600.

39 R. Swaminathn, V. K. Ravi, S. Kumar, M. V. Kumar and

N. Chandra, Adv. Protein Chem. Struct. Biol., 2011, 84, 63-111. 40 G. Merlini and V. Bellotti, Clin. Chim. Acta, 2005, 357, 168-172.

41 M. B. Pepys, P. N. Hawkins, D. R. Booth, D. M. Vigushin,

G. A. Tennent, A. K. Soutar, N. Totty, O. Nguyen, C. C. F. Blake, C. J. Terry, T. G. Feest, A. M. Zalin and J. J. Hsuan, Nature, 1993, 363, 553-557.

42 D. R. Booth, M. Sunde, V. Bellotti, C. V. Robinson, W. L. Hutchinson, P. A. Fraser, P. N. Hawkins, C. M. Dobson, S. E. Radford, C. C. F. Blake and M. R. Pepys, Nature, 1997, 385, 787-793.

43 P. M. Boyer and J. T. Hsu, Chem. Eng. Sci., 1992, 47, 241-251. 44 J. R. Hobbs and A. D. Morgan, J. Pathol. Bacteriol., 1963, 86, 437-442.

45 S. M. Saeed and G. Fine, Am. J. Clin. Pathol., 1967, 47, 588593.

46 J. Wall, C. Murphy and L. A. Solomon, Methods Enzymol., 1999a, 309, 204-217.

47 H. Levine, Protein Sci., 1993, 2, 404-410.

48 L. Nielsen, R. Khurana, A. Coats, S. Frokjaer, J. Brange, S. Vyas, V. N. Uversky and A. L. Fink, Biochemistry, 2001, 40, 6036-6046.

49 M. Ohi, Y. Li, Y. Cheng and T. Walz, Biol. Proced. Online, 2004, 6, 23-34.

50 E. A. Varuvel and M. Jeyaraj, Int. J. Pharm. Pharm. Sci., 2014, 6, 123-126.

51 R. Shah, V. Gulati and E. A. Palombo, Phytother. Res., 2012, 26, 1594-1605.

52 B. Krishnamachary, K. P. Arun, B. Pemiah, S. Krishnaswamy, U. M. Krishnan, S. Sethuraman and R. K. Sekar, J. Chem., 2013, 2013, 1-8.

53 W. F. Xue, S. W. Homans and S. E. Radfor, Proc. Natl. Acad. Sci. U. S. A., 2008, 105, 8926-8931.

54 W. E. Klunk, R. F. Jacob and R. P. Manson, Methods Enzymol., 1999, 309, 285-305.

55 Y. Yoshimura, Y. Lin, H. Yagi, Y. H. Lee, H. Kitayama, K. Sakurai, M. So, H. Ogi, H. Naiki and Y. Goto, Proc. Natl. Acad. Sci. U. S. A., 2012, 109, 14446-14451.

56 H. J. Kim, J. Kim, K. S. Kang, K. T. Lee and H. O. Yang, Biomol. Ther., 2014, 22, 275-281.

57 A. Sarkaki, Y. Farbood, M. Dolatshahi, S. M. T. Mansouri and A. Khodadadi, Acta Med. Iran., 2016, 54, 494-502.

58 M. I. Naseer, H. Y. Lee and M. O. Kim, Synapse, 2010, 64, 467-477. 